\title{
Dynamics of a Diffusive Multigroup SVIR Model with Nonlinear Incidence
}

\author{
Jinhu Xu $\mathbb{D}^{1}$ and Yan Geng ${ }^{2}$ \\ ${ }^{1}$ School of Sciences, Xi'an University of Technology, Xi'an 710048, China \\ ${ }^{2}$ School of Science, Xi'an Polytechnic University, Xi'an 710048, China \\ Correspondence should be addressed to Jinhu Xu; xujinhu09@163.com
}

Received 22 August 2020; Revised 25 October 2020; Accepted 4 November 2020; Published 9 December 2020

Academic Editor: Dimitri Volchenkov

Copyright ( 2020 Jinhu Xu and Yan Geng. This is an open access article distributed under the Creative Commons Attribution License, which permits unrestricted use, distribution, and reproduction in any medium, provided the original work is properly cited.

\begin{abstract}
In this paper, a multigroup SVIR epidemic model with reaction-diffusion and nonlinear incidence is investigated. We first establish the well-posedness of the model. Then, the basic reproduction number $\mathfrak{R}_{0}$ is established and shown as a threshold: the disease-free steady state is globally asymptotically stable if $\mathfrak{R}_{0}<1$, while the disease will be persistent when $\mathfrak{R}_{0}>1$. Moreover, applying the classical method of Lyapunov and a recently developed graph-theoretic approach, we established the global stability of the endemic equilibria for a special case.
\end{abstract}

\section{Introduction}

As is well-known, mathematical models have played a key role in describing the dynamical evolution of infectious diseases [1-10]. For example, during the special battle for preventing the novel coronavirus (COVID-19) spreading, not only medical and biological research but also theoretical studies based on mathematical modeling may play a crucial role in analyzing and forecasting the spreading of the disease (see, for example, [4-10] and references therein; also, some useful advices for controlling the disease spreading have been given by the researchers).

The spatial heterogeneity has been regarded as an important role that affects the spatial spreading of disease. Therefore, complex models are needed to investigate the spread of diseases. In recent years, reaction-diffusion models involving environmental heterogeneity have been proposed and studied to find reliable measures to control disease spreading [11-22]. In particular, the basic reproduction number is an important threshold value for investigation of the dynamics of epidemic models. Then, Wang and Zhao [14] defined the basic reproduction number and obtained its computation formula for general reaction-diffusion epidemic models. Xu et al. [21] studied an SVIR epidemic model with reaction-diffusion and the existences of travelling waves were investigated, while Zhang and Liu [22] also studied the existence of travelling waves for an SVIR epidemic model but with nonlocal dispersal and time delay.

Notice that the essential assumption in classical compartmental epidemic models is that the individuals are homogeneously mixed, which means each individual has the same probability to get infected. However, infected probability may be different for each individual in terms of the impact of different factors, such as education levels, age, gender, and communities. To overcome this problem, multigroup models have been proposed by many researchers by dividing the individuals into different groups and most of these work focus on the global dynamics of the models (see, for example, [23-30]). Particularly, one of the earliest works of multigroup models was proposed by Lajmanovich and York [23] when studied the gonorrhea disease transmission in a nonhomogeneous population. In order to investigate the global dynamics of multigroup models, a subtle grouping method in estimating the derivatives of Lyapunov functionals guided by graph theory for multigroup models was developed in [28-30]. For example, Kuniya [25] considered a multigroup SVIR epidemic model with vaccination and the global stability of endemic equilibria were established by the 
method of Lyapunov function. However, spatially heterogeneous was not considered in the model in [25]. To the best of our knowledge, there are few results of multigroup SVIR epidemic model with reaction-diffusion. The existing results are focusing on SIS and SIR models (see [31-34]). On the contrary, incidence rate also plays an important role in modeling the epidemic models, which has been frequently used to describe the nature of certain phenomena and obtained much exact results. In addition, applying general incidence rates can obtain the unification theory by the omission of unessential detail, see, for example, [21, 35-37] and references therein. Hence, inspired by [21, 25] and the above considerations, in this paper, we consider the following multigroup SVIR epidemic model with reactiondiffusion and general incidence rate:

$$
\begin{cases}\frac{\partial S_{i}}{\partial t}=\nabla \cdot\left(d_{1 i}(x) \nabla S_{i}\right) \lambda_{i}(x)-\left(\mu_{i}^{S}(x)+\xi_{i}(x)\right) S_{i}-\sum_{j=1}^{n} \beta_{i j}(x) S_{i} f_{j}\left(I_{j}\right), & t \geq 0, x \in \Omega, 1 \leq i \leq n \\ \frac{\partial V_{i}}{\partial t}=\nabla \cdot\left(d_{2 i}(x) \nabla V_{i}\right)+\xi_{i}(x) S_{i}-\sum_{j=1}^{n} \tilde{\beta}_{i j}(x) V_{i} f_{j}\left(I_{j}\right)-\mu_{i}^{V}(x) V_{i}, & t \geq 0, x \in \Omega, 1 \leq i \leq n \\ \frac{\partial I_{i}}{\partial t}=\nabla \cdot\left(d_{3 i}(x) \nabla I_{i}\right)+\sum_{j=1}^{n}\left(\beta_{i j}(x) S_{i}+\widetilde{\beta}_{i j}(x) V_{i}\right) f_{j}\left(I_{j}\right)-\left(\mu_{i}^{I}(x)+\delta_{i}(x)+\gamma_{i}(x)\right) I_{i}, & t \geq 0, x \in \partial \Omega, 1 \leq i \leq n, \\ \frac{\partial R_{i}}{\partial t}=\nabla \cdot\left(d_{4 i}(x) \nabla R_{i}\right)+\gamma_{i}(x) I_{i}-\mu_{i}^{R}(x) R_{i}, & t \geq 0, x \in \Omega, 1 \leq i \leq n\end{cases}
$$

with the homogeneous Neumann boundary conditions

$$
\frac{\partial S_{i}}{\partial \nu}=\frac{\partial V_{i}}{\partial v}=\frac{\partial I_{i}}{\partial \nu}=\frac{\partial R_{i}}{\partial v}=0, \quad t \geq 0, x \in \partial \Omega, 1 \leq i \leq n,
$$

and the initial conditions

$$
\left(S_{i}(0, x), V_{i}(0, x), I_{i}(0, x), R_{i}(0, x)\right)=\left(\phi_{1 i}(x), \phi_{2 i}(x), \phi_{3 i}(x), \phi_{4 i}(x)\right)
$$

for $x \in \Omega, 1 \leq i \leq n$, where $\Omega$ is a domain in $\mathbb{R}^{n}$ with smooth boundary $\Omega$ and $v$ is the outward normal vector to the boundary $\partial \Omega$. Initial functions $\phi_{k i}(x)(k=1,2,3,4,1 \leq$ $i \leq n)$ are nonnegative and continuously defined on $\bar{\Omega}$. $S_{i}=S_{i}(t, x), V_{i}=V_{i}(t, x), I_{i}=I_{i}(t, x)$, and $R_{i}=R_{i}(t, x)$ stand for the densities of the susceptible, vaccinated, infective, and recovered individuals in the $i$-th group at time $t$ and spatial location $x$, respectively. $\lambda_{i}(x)$ is the input rate of $S_{i}$ in spatial location $x ; \mu_{i}^{S}(x), \mu_{i}^{V}(x), \mu_{i}^{I}(x)$, and $\mu_{i}^{R}(x)$ denote the natural death rates of $S_{i}, V_{i}, I_{i}$, and $R_{i}$ in spatial location $x$, respectively; $\delta_{i}(x)$ is the death rate induced by the disease in spatial location $x ; \xi_{i}(x)$ is the vaccination rate of $S_{i}$ in spatial location $x ; \gamma_{i}(x)$ is the rate of recovery from infection in spatial location $x ; \beta_{i j}(x)$ is the infection rate of $S_{i}$ infected by $I_{j}$ in spatial location $x ; \widetilde{\beta}_{i j}(x)$ is the infection rate of $V_{i}$ infected by $I_{j}$ in spatial location $x$; and $d_{1 i}(x), d_{2 i}(x)$, $d_{3 i}(x)$, and $d_{4 i}(x)$ are the diffusion rate of $S_{i}, V_{i}, I_{i}$, and $R_{i}$ in spatial location $x$, respectively. All location-dependent parameters are continuous and strictly positive defined on $\bar{\Omega}$, and $f_{j}\left(I_{j}\right)$ denotes the force of infection. We assume the function $f_{j}\left(I_{j}\right)$ satisfies the following properties:

$$
\begin{array}{cl}
f_{j}(0)=0, & f_{j}\left(I_{j}\right)>0 \text { for } I_{j}>0, \\
f_{j}^{\prime}\left(I_{j}\right)>0, & f_{j}^{\prime \prime}\left(I_{j}\right) \leq 0 \text { for all } I_{j}>0 .
\end{array}
$$

It is natural to assume that $f_{j}(0)=0$ due to the fact that disease cannot spread if there is no infection. The disease spreads heavily with the increasing number of infected individuals; thus, it reasonable to suppose $f_{j}^{\prime}\left(I_{j}\right)>0$. Since the infectivity of infected individuals cannot be unbounded, it should reach a certain level when the infected individuals are heavy. Therefore, the assumption $f_{j}^{\prime \prime}\left(I_{j}\right) \leq 0$ implies that there exists a peak level for the infectivity of the infected individuals at some certain time. Similar to $[25,34]$, we also assume the $n$-square matrix $\left(\beta_{i j}(x)\right)_{n \times n}$ is nonnegative and irreducible.

Because the last equation of model (1) is decoupled from other equations, we indeed need to study the following subsystem of (1): 


$$
\begin{cases}\frac{\partial S_{i}}{\partial t}=\nabla \cdot\left(d_{1 i}(x) \nabla S_{i}\right)+\lambda_{i}(x)-\left(\mu_{i}^{S}(x)+\xi_{i}(x)\right) S_{i}-\sum_{j=1}^{n} \beta_{i j}(x) S_{i} f_{j}\left(I_{j}\right), & t \geq 0, x \in \Omega, 1 \leq i \leq n, \\ \frac{\partial V_{i}}{\partial t}=\nabla \cdot\left(d_{2 i}(x) \nabla V_{i}\right)+\xi_{i}(x) S_{i}-\sum_{j=1}^{n} \widetilde{\beta}_{i j}(x) V_{i} f_{j}\left(I_{j}\right)-\mu_{i}^{V}(x) V_{i}, & t \geq 0, x \in \Omega, 1 \leq i \leq n, \\ \frac{\partial I_{i}}{\partial t}=\nabla \cdot\left(d_{3 i}(x) \nabla I_{i}\right)+\sum_{j=1}^{n}\left(\beta_{i j}(x) S_{i}+\widetilde{\beta}_{i j}(x) V_{i}\right) f_{j}\left(I_{j}\right)-\left(\mu_{i}^{I}(x)+\delta_{i}(x)+\gamma_{i}(x) I_{i}\right), & t \geq 0, x \in \Omega, 1 \leq i \leq n .\end{cases}
$$

The rest of this paper is organized as follows. In Section 2 , some preliminaries are introduced for the well-posedness of the model. In Section 3, we define the basic reproduction number $\mathfrak{R}_{0}$. In Section 4, the threshold dynamics are established in terms of $\boldsymbol{R}_{0}$. An special case is performed as a supplementary to the theoretical results in Section 5. A brief conclusion ends the paper.

\section{Well-Posedness}

Throughout this paper, we denote $\bar{f}=\max _{x \in \bar{\Omega}} f(x)$ and $f=\min _{x \in \bar{\Omega}} f(x)$. Let $\mathbb{Y}=C\left(\bar{\Omega}, \mathbb{R}^{n}\right)$ with the norm $\|\cdot\|_{\mathbb{Y}}$ and $\mathbb{Y}^{+}=C\left(\Omega, \mathbb{R}_{+}^{n}\right)$. It is easy to see that $\left(\mathbb{Y}, \mathbb{Y}^{+}\right)$is a strongly ordered Banach space. Let $\mathbb{X}=\mathbb{Y} \times \mathbb{Y} \times \mathbb{Y}$ with norm $\|\phi\|_{\mathbb{X}}=\max \left\{\left\|\phi_{1}\right\|_{\mathbb{Y}},\left\|\phi_{2}\right\|_{\mathbb{Y}},\left\|\phi_{3}\right\|_{\mathbb{Y}}\right\}$, where $\quad \phi=\left(\phi_{1}, \phi_{2}, \phi_{3}\right)$ $\in \mathbb{X}, \phi_{i} \in \mathbb{Y}$. Denote $\mathbb{X}^{+}=\mathbb{Y}^{+} \times \mathbb{Y}^{+} \times \mathbb{Y}^{+}$be the positive cone of $\mathbb{X}$. For convenience, set $\phi_{k}=\left(\phi_{k 1}, \phi_{k 1}, \ldots, \phi_{k 1}\right)$ for $k=1,2,3 . \quad S=\left(S_{1}, S_{2}, \ldots, S_{n}\right), V=\left(V_{1}, V_{2}, \ldots, V_{n}\right)$, and $I=\left(I_{1}, I_{2}, \ldots, I_{n}\right)$. Denote

$$
T_{1 i}, T_{2 i}, T_{3 i}: C(\bar{\Omega}, \mathbb{R}) \longrightarrow C(\bar{\Omega}, \mathbb{R})
$$

be the $C_{0}$ semigroup associated with $\nabla \cdot\left(d_{1 i}(x) \nabla\right)-$ $\left(\mu_{i}^{S}(x)+\xi_{i}(x)\right), \quad \nabla \cdot\left(d_{2 i}(x) \nabla\right)-\mu_{i}^{V}(x)$, and $\nabla \cdot\left(d_{3 i}(x)\right.$ $\nabla)-\left(\mu_{i}^{I}(x)+\delta_{i}(x)+\gamma_{i}(x)\right)$, respectively, subject to the Neumann boundary condition. Let $\Gamma_{k i}(t, x, y)$ be the Green function associated with $\nabla \cdot\left(d_{1 i}(x) \nabla\right)-\left(\mu_{i}^{S}(x)+\xi_{i}(x)\right)$, $\nabla \cdot\left(d_{2 i}(x) \nabla\right)-\mu_{i}^{V}(x)$, and $\nabla \cdot\left(d_{3 i}(x) \nabla\right)-\left(\mu_{i}^{I}(x)+\delta_{i}\right.$ $\left.(x)+\gamma_{i}(x)\right)$, respectively, subject to the Neumann boundary condition. Then, for any $\phi \in C(\bar{\Omega}, \mathbb{R})$ and $t>0$, we have

$$
\left(T_{k i}(t) \phi\right)(x)=\int_{\Omega} \Gamma_{k i}(t, x, y) \phi(y) \mathrm{d} y, \quad k=1,2,3,1 \leq i \leq n .
$$

Applying Corollary 7.2.3 in [38], we know that, for each $t>0, T_{k i}(t): C(\bar{\Omega}, \mathbb{R}) \longrightarrow C(\bar{\Omega}, \mathbb{R})$ is compact and strongly positive. Then, there exist constants $A_{k i}>0(k=1,2,3)$, satisfying $\left\|T_{k i}(t)\right\| \leq A_{k i} e^{\alpha_{k i} t}$ for each $t \geq 0$, where $\alpha_{k i}$ denotes the principle eigenvalue of $\nabla \cdot\left(d_{1 i}(x) \nabla\right)-\left(\mu_{i}^{S}(x)+\xi_{i}(x)\right)$, $\nabla \cdot\left(d_{2 i}(x) \nabla\right)-\mu_{i}^{V}(x)$, and $\nabla \cdot\left(d_{3 i}(x) \nabla\right)-\left(\mu_{i}^{I}(x)+\right.$ $\left.\delta_{i}(x)+\gamma_{i}(x)\right)$ subject to the Neumann boundary condition. Define

$$
\begin{aligned}
& F_{1 i}(\phi)(x)=\lambda_{i}(x)-\sum_{j=1}^{n} \beta_{i j}(x) \phi_{1 i}(x) f_{j}\left(\phi_{3 j}(x)\right), 1 \leq i \leq n, \\
& F_{2 i}(\phi)(x)=\xi_{i}(x) \phi_{1 i}(x)-\sum_{j=1}^{n} \widetilde{\beta}_{i j}(x) \phi_{2 i}(x) f_{j}\left(\phi_{3 j}(x)\right), 1 \leq i \leq n, \\
& F_{3 i}(\phi)(x)=\sum_{j=1}^{n} \beta_{i j}(x) \phi_{1 i}(x) f_{j}\left(\phi_{3 j}(x)\right)+\sum_{j=1}^{n} \tilde{\beta}_{i j}(x) \phi_{2 i}(x) f_{j}\left(\phi_{3 j}(x)\right), 1 \leq i \leq n,
\end{aligned}
$$

for $t \geq 0, x \in \bar{\Omega}$, and $\phi=\left(\phi_{1}, \phi_{2}, \phi_{3}\right) \in \mathbb{X}^{+}$. Set $u(t, \cdot, \phi)=$ $(S(t, \cdot, \phi), V(t, \cdot, \phi), I(t, \cdot, \phi))$ be the solution of model (5) with initial function $\phi=\left(\phi_{1}, \phi_{2}, \phi_{3}\right) \in \mathbb{X}^{+}$, and then, model (5) can be rewritten as

$$
\begin{aligned}
& S_{i}(t, \cdot, \phi)=T_{1 i}(t) \phi_{1}+\int_{0}^{t} T_{1 i}(t-s) F_{1 i}(u(s, \cdot, \phi)) \mathrm{d} s, \\
& V_{i}(t, \cdot, \phi)=T_{2 i}(t) \phi_{2}+\int_{0}^{t} T_{2 i}(t-s) F_{2 i}(u(s, \cdot, \phi)) \mathrm{d} s, \\
& I_{i}(t, \cdot, \phi)=T_{3 i}(t) \phi_{3}+\int_{0}^{t} T_{3 i}(t-s) F_{3 i}(u(s, \cdot, \phi)) \mathrm{d} s,
\end{aligned}
$$

for $t>0$ and $1 \leq i \leq n$. By virtue of Corollary 4 in [39], we have the following.

Lemma 1. For $\phi=\left(\phi_{1}, \phi_{2}, \phi_{3}\right) \in \mathbb{X}^{+}$, model (5) has a unique mild solution $u(t, \cdot, \phi)=(S(t, \cdot, \phi), V(t, \cdot, \phi), I(t, \cdot, \phi)) \in \mathbb{X}^{+}$ on $\left[0, \tau_{\infty}\right)$ and $\tau_{\infty} \leq \infty$. Moreover, this solution is a classical solution.

Then, we give the existence of solutions of model (5).

Theorem 1. The model (5) has a unique solution $u(t, \cdot, \phi)=$ $(S(t, \cdot, \phi), V(t, \cdot, \phi), I(t, \cdot, \phi)) \in \mathbb{X}^{+} \quad$ on $\quad[0, \infty)$ with 
$\phi=\left(\phi_{1}, \phi_{2}, \phi_{3}\right) \in \mathbb{X}^{+}$. Furthermore, the solution semiflow $\Phi(t)=u(t, \cdot): \mathbb{X}^{+} \longrightarrow \mathbb{X}^{+}$of model (5) defined by

$$
\Phi(t) \phi=u(t, \cdot, \phi), \quad t \geq 0,
$$

admits a global compact attractor.

Proof. Suppose to the contrary that $\tau_{\infty}<\infty$, then $\|u(t, \cdot, \phi)\| \longrightarrow+\infty$ as $t \longrightarrow \tau_{\infty}$ by Theorem 2 in [39]. It follows from the first equation of model (5) that

$$
\begin{aligned}
& \frac{\partial S_{i}}{\partial t} \leq \nabla \cdot\left(d_{1 i}(x) \nabla S_{i}\right)+\bar{\lambda}_{i}-\left(\underline{-}_{{ }_{i}}{ }^{S}+\underline{-}_{i}\right) S_{i}, \\
& t \in\left[0, \tau_{\infty}\right), x \in \Omega, 1 \leq i \leq n .
\end{aligned}
$$

By the comparison principle and Lemma 2 in [40], there exists a constant $\mathscr{M}_{1}>0$ such that $S_{i}(t, x) \leq \mathscr{M}_{1}(1 \leq i \leq n)$ for $t \in\left[0, \tau_{\infty}\right), x \in \bar{\Omega}$. Furthermore, similar procedure can be applied to the second equation of model (5), and then, there exists a constant $\mathscr{M}_{2}>0$ such that $V_{i}(t, x) \leq \mathscr{M}_{2}$ $(1 \leq i \leq n)$ for $t \in\left[0, \tau_{\infty}\right), x \in \bar{\Omega}$. Hence, from the third equation of model (5), we have

$$
\begin{aligned}
& \frac{\partial I_{i}}{\partial t} \leq \nabla \cdot\left(d_{3 i}(x) \nabla I_{i}\right)+\sum_{j=1}^{n}\left(\bar{\beta}_{i j} \mathscr{M}_{1}+\overline{\widetilde{\beta}}_{i j} \mathscr{M}_{2}\right) f_{j}^{\prime}(0) I_{j} \\
& -\left(\underset{-i}{\mu}+\underline{-}_{i}+\underset{{ }_{i}}{\mu_{i}}\right) I_{i} .
\end{aligned}
$$

Now, we consider the following comparison system:

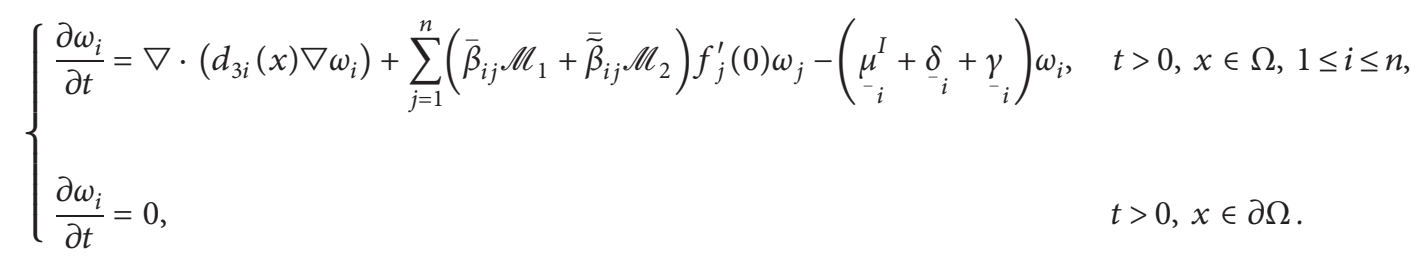

It follows from the standard Krein-Rutman theorem (see [41]) that the eigenvalue problem of system (13) admits a principle eigenvalue $\lambda$ with a strongly positive eigenfunction $\hat{\phi}_{2}=\left(\hat{\phi}_{21}, \hat{\phi}_{22}, \ldots, \hat{\phi}_{2 n}\right)$. Thus, system (13) has a solution $\varsigma e^{\lambda t} \hat{\phi}_{2}(x)$ for $t \geq 0$, where $\varsigma$ is a positive constant, satisfying $\varsigma \hat{\phi}_{2} \geq\left(I_{1}(0, x), I_{2}(0, x), \ldots, I_{n}(0, x)\right)$ for $x \in \bar{\Omega}$. By using the comparison principle, we have

$$
\begin{array}{r}
\left(I_{1}(t, x), I_{2}(t, x), \ldots, I_{n}(t, x)\right) \leq \varsigma e^{\lambda t} \hat{\phi}_{2}(x), \\
t \in\left[0, \tau_{\infty}\right), x \in \bar{\Omega}, 1 \leq i \leq n .
\end{array}
$$

Thus, there exists a constant $\mathscr{M}_{3}$ such that

$$
I_{i}(t, x) \leq \mathscr{M}_{3}, \quad x \in \bar{\Omega}, 1 \leq i \leq n,
$$

which leads to a contradiction. Hence, the global existence of $u(t, \cdot, \phi)$ is derived.

Now, we are in the position to show the solution is also ultimately bounded. Indeed, it follows from the comparison principle, (11), and Lemma 2 in [40] that there exist $t_{1}>0$ and $\mathscr{A}_{1}>0$ such that $S_{i}(t, x) \leq \mathscr{A}_{1}, t \geq t_{1}, \forall x \in \bar{\Omega}$. Moreover, from the second equation of model (5) and applying similar procedures, we can find $\mathscr{A}_{2}>0$ and $t_{2}>0$, satisfying $V_{i}(t, x) \leq \mathscr{A}_{2}$ for $t \geq t_{2}, \forall x \in \bar{\Omega}$.

Denote

$$
Q_{i}(t)=\int_{\Omega}\left(S_{i}(t, x)+V_{i}(t, x)+I_{i}(t, x) \mathrm{d} x\right), \quad 1 \leq i \leq n .
$$

Then, we have

$$
\begin{aligned}
\frac{\mathrm{d} Q_{i}}{\mathrm{~d} t} & =\int_{\Omega}\left(\lambda_{i}(x)-\mu_{i}^{S}(x) S_{i}(t, x)-\mu_{i}^{V}(x) V(t, x)-\left(\mu_{i}^{I}(x)+\delta_{i}(x)+\gamma_{i}(x)\right) I_{i}(t, x)\right) \mathrm{d} x \\
& \leq \int_{\Omega} \lambda_{i}(x) \mathrm{d} x-\min _{x \in \bar{\Omega}}\left\{\begin{array}{c}
\mu^{S}, \mu^{V}, \mu_{i}{ }^{I}+{ }_{-}{ }_{i}+{ }_{i} \\
{ }_{i}
\end{array}\right\} Q_{i}, \quad t \geq 0 .
\end{aligned}
$$

Thus, there exist $t_{3}>0$ and $\mathscr{A}_{3}>0$ such that $Q_{i}(t) \leq \mathscr{A}_{3}(1 \leq i \leq n)$ for any $t \geq t_{3}$. Next, we denote $\tau_{j}^{i}$ be the eigenvalue of $\nabla \cdot\left(d_{3 i}(x) \nabla\right)-\left(\mu_{i}^{I}(x)+\delta_{i}(x)+\gamma_{i}(x)\right)$ subject to the Neumann boundary condition with eigenfunction $\varphi_{j}^{i}(x)$, which satisfies $\tau_{1}^{i}>\tau_{2}^{i} \geq \tau_{3}^{i} \geq \cdots \tau_{j}^{i} \geq \cdots$. From Chapter 5 in [42], one obtains

$$
\Gamma_{3 i}(t, x, y)=\sum_{j \geq 1} e^{\tau_{j}^{i} t} \varphi_{j}^{i}(x) \varphi_{j}^{i}(y), \quad 1 \leq i \leq n .
$$

Since $\varphi_{j}^{i}(x)$ is uniformly bounded, there exists constant $\kappa_{3 i}>0$ such that $\Gamma_{3 i}(t, x, y) \leq \kappa_{3 i} \sum_{j \geq 1} e^{\tau_{j}^{i} t}$ for $t>0$. Moreover,

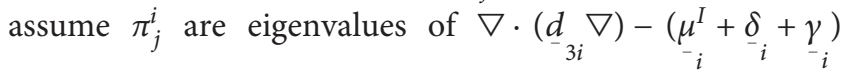


subject to Neumann boundary condition, which satisfies $\left.\pi_{1}^{i}=-\underset{-i}{\left(\mu^{I}\right.}+\underset{i}{\delta_{i}}+\underset{i}{\gamma}\right)>\pi_{2}^{i} \geq \pi_{3}^{i} \geq \cdots \pi_{j}^{i} \geq \cdots$. By Theorem 2.4.7 in Wang [43], one gets $\pi_{j}^{i} \geq \tau_{j}^{i}$ for all $j \in \mathbb{N}_{+}$. Since $\pi_{j}^{i}$ decreases like $-n^{2}$, there exists $\kappa_{3}>0$ such that

$$
\Gamma_{3 i} \leq \kappa_{3 i} \sum_{j \geq 1} e^{\pi_{j}^{i} t} \leq \kappa_{3} e^{\pi_{1}^{i} t}=\kappa_{3} e^{-\left(\begin{array}{c}
\mu^{I}+\underline{\delta}_{i}+\underline{\gamma}_{i} \\
{ }_{i}
\end{array}\right) t}, \quad \forall t>0 .
$$

Let $\hat{t}=\max \left\{t_{1}, t_{2}, t_{3}\right\}$. For all $t \geq \hat{t}$, by (9) and (4), we have

$$
\begin{aligned}
& I_{i}(t, x)=T_{3 i}(t) I_{i}(\hat{t}, x)+\int_{\widehat{t}}^{t} T_{3 i}(t-s)\left[\sum_{j=1}^{n}\left(\beta_{i j}(x) S_{i}(s, x)+\tilde{\beta}_{i j}(x) V_{i}(s, x)\right) f_{j}\left(I_{j}(s, x)\right)\right] \mathrm{d} s \\
& \leq A_{3 i} e^{\alpha_{3 i}(t-\hat{t})}\left\|I_{i}(\hat{t}, x)\right\|+\int_{\hat{t}}^{t} \int_{\Omega} \Gamma_{3 i}(t-s, x, y)\left[\sum_{j=1}^{n}\left(\beta_{i j}(y) S_{i}(s, y)+\widetilde{\beta}_{i j}(y) V_{i}(s, y)\right) \times f_{j}^{\prime}(0) I_{j}(s, y)\right] \mathrm{d} y \mathrm{~d} s
\end{aligned}
$$

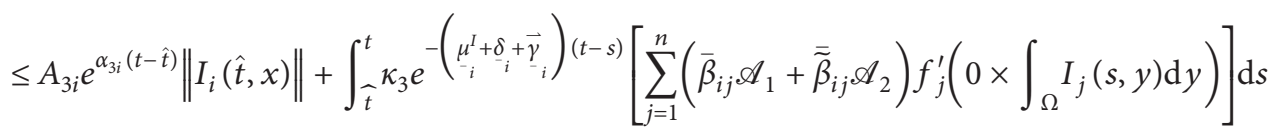

$$
\begin{aligned}
& \leq A_{3 i} e^{\alpha_{3 i}(t-\hat{t})}\left\|I_{i}(\hat{t}, x)\right\|+\kappa_{3} \mathscr{A}_{3} \int_{\hat{t}}^{t} e^{-\left(\mu_{i}^{I}+\hat{\delta}_{i}+\vec{\gamma}_{i}\right)(t-s)} \cdot \sum_{j=1}^{n}\left(\bar{\beta}_{i j} \mathscr{A}_{1}+\overline{\tilde{\beta}}_{i j} \mathscr{A}_{2}\right) f_{j}^{\prime}(0) \mathrm{d} s \\
& =A_{3 i} e^{\alpha_{3 i}(t-\hat{t})}\left\|I_{i}(\hat{t}, x)\right\|+\kappa_{3} \mathscr{A}_{3} \sum_{j=1}^{n}\left(\bar{\beta}_{i j} \mathscr{A}_{1}+\overline{\tilde{\beta}}_{i j} \mathscr{A}_{2}\right) f_{j}^{\prime}(0) \frac{1-e^{-\left(\mu_{i}^{I}+\underline{x}_{i}+\vec{\gamma}_{i}\right)(t-\hat{t})}}{\underline{\mu}^{I}+\underline{i}_{i}+\vec{\gamma}_{i}} \\
& \leq A_{3 i} e^{\alpha_{3 i}(t-\hat{t})}\left\|I_{i}(\hat{t}, x)\right\|+\frac{\kappa_{3} \mathscr{A}_{3} \sum_{j=1}^{n}\left(\bar{\beta}_{i j} \mathscr{A}_{1}+\overline{\tilde{\beta}}_{i j} \mathscr{A}_{2}\right) f_{j}^{\prime}(0)}{\underline{\mu}_{i}^{I}+\underline{-}_{i}+\underline{\underline{\gamma}}_{i}},
\end{aligned}
$$

which yields that

$$
\limsup _{\mathrm{t} \rightarrow \infty}\left\|I_{i}(t, x)\right\| \leq \frac{\kappa_{3} \mathscr{A}_{3} \sum_{j=1}^{n}\left(\bar{\beta}_{i j} \mathscr{A}_{1}+\overline{\tilde{\beta}}_{i j} \mathscr{A}_{2}\right) f_{j}^{\prime}(0)}{\underline{\mu}_{-}^{\mathrm{I}}+\underline{\delta}_{i}+\underline{\underline{\gamma}}_{i}}, \quad 1 \leq i \leq n .
$$

Thus, the above discussion implies that the system (5) is point dissipative. Furthermore, by Theorem 2.2.6 in [44], the solution semiflow $\Phi(t)$ is compact for any $t>0$. Therefore, it follows from Theorem 3.4.8 in [45] that $\Phi(t)$ has a global compact attractor in $\mathbb{X}^{+}$.

\section{Basic Reproduction Number}

For each $1 \leq i \leq n$, consider the following subsystem of model (5):

$$
\left\{\begin{array}{l}
\frac{\partial S_{i}}{\partial t}=\nabla \cdot\left(d_{1 i}(x) \nabla S_{i}\right)+\lambda_{i}(x)-\left(\mu_{i}^{S}(x)+\xi_{i}(x)\right) S_{i}, \quad t>0, x \in \Omega \\
\frac{\partial V_{i}}{\partial t}=\nabla \cdot\left(d_{2 i}(x) \nabla V_{i}\right)+\xi_{i}(x) S_{i}-\mu_{i}^{V}(x) V_{i}, \quad t>0, x \in \Omega \\
\frac{\partial S_{i}}{\partial \nu}=\frac{\partial V_{i}}{\partial \nu}=0, \quad t>0, x \in \partial \Omega .
\end{array}\right.
$$

It follows from the Lemma 2.2 in [40] that the following system 


$$
\begin{cases}\frac{\partial S_{i}}{\partial t}=\nabla \cdot\left(d_{1 i}(x) \nabla S_{i}\right)+\lambda_{i}(x)-\left(\mu_{i}^{S}(x)+\xi_{i}(x)\right) S_{i}, & t>0, x \in \Omega, \\ \frac{\partial S_{i}}{\partial \nu}=0, & t>0, x \in \partial \Omega,\end{cases}
$$

admits a unique positive steady state $S_{i}^{0}(x)$ which satisfies the equation

$$
\nabla \cdot\left(d_{1 i}(x) \nabla S_{i}^{0}(x)\right)+\lambda_{i}(x)-\left(\mu_{i}^{S}(x)+\xi_{i}(x)\right) S_{i}^{0}(x)=0
$$

with $\partial S_{i}^{0}(x) / \partial \nu=0$ for $x \in \partial \Omega$, which is globally asymptotically stable in $C\left(\bar{\Omega}, \mathbb{R}_{+}\right)$. Hence, the second equation of system (22) is asymptotic to

$$
\frac{\partial V_{i}}{\partial t}=\nabla \cdot\left(d_{2 i}(x) \nabla V_{i}\right)+\xi_{i}(x) S_{i}^{0}(x)-\mu_{i}^{V}(x) V_{i}
$$

By Lemma 2.2 in [40] and Corollary 4.3 [46], there exists a globally asymptotically stable steady state $V_{i}^{0}(x)$. Therefore, concluding from the above discussion, we know that model (5) admits a unique disease-free steady state $E_{0}(x)=$ $\left(S^{0}(x), V^{0}(x), 0\right)$ with $S^{0}(x)=\left(S_{1}^{0}(x), S_{2}^{0}(x), \ldots, S_{n}^{0}(x)\right)$, $V^{0}(x)=\left(V_{1}^{0}(x), V_{2}^{0}(x), \ldots, V_{n}^{0}(x)\right)$, and $0=(0,0, \ldots, 0)$. Furthermore, if all the parameters of model (5) are positive constants, then we have $S_{i}^{0}(x)=\lambda_{i} / \mu_{i}^{S}+\xi_{i}$ and $V_{i}^{0}(x)=\lambda_{i} \xi_{i} / \mu_{i}^{V}\left(\mu_{i}^{S}+\xi_{i}\right)(1 \leq i \leq n)$.

Linearizing model (5) at $E_{0}$, we obtain the linearized system

$$
\begin{cases}\frac{\partial I_{i}}{\partial t}=\nabla \cdot\left(d_{3 i}(x) \nabla I_{i}\right)+\sum_{j=1}^{n} \theta_{i j}(x) f_{j}^{\prime}(0) I_{j}-\left(\mu_{i}^{I}(x)+\delta_{i}(x)+\gamma_{i}(x)\right) I_{i}, & t>0, x \in \Omega, 1 \leq i \leq n, \\ \frac{\partial I_{i}}{\partial \nu}=0, & t>0, x \in \partial \Omega, 1 \leq i \leq n .\end{cases}
$$

Substituting $I_{i}(t, x)=e^{\lambda t} \phi_{3 i}(x)$ into (26), we obtain

$$
\begin{cases}\lambda \phi_{3 i}(x)=\nabla \cdot\left(d_{3 i}(x) \nabla \phi_{3 i}(x)\right)+\sum_{j=1}^{n} \theta_{i j}(x) f_{j}^{\prime}(0) \phi_{3 i}(x)-\left(\mu_{i}^{I}(x)+\delta_{i}(x)+\gamma_{i}(x)\right) \phi_{3 i}(x), & t>0, x \in \Omega, 1 \leq i \leq n \\ \frac{\partial \phi_{3 i}=0,}{\partial \nu} & t>0, x \in \partial \Omega, 1 \leq i \leq n\end{cases}
$$

From Theorem 7.6.1 in [38], we have the following result.

Lemma 2. The eigenvalue problem (27) admits a principle eigenvalue $\lambda_{0}$ with a strictly positive eigenfunction.

Denote $T_{3}=\left(T_{31}, T_{32}, \ldots, T_{3 n}\right)$. Assume that the distribution of initial infection is $\phi_{3}(x) \in \mathbb{Y}_{+}$. Then, $\mathscr{F}(x) T_{3} \phi_{3}(x)$ is the distribution of new infective part as time evolves. Therefore, we use

$$
\mathscr{L}\left(\phi_{3}\right)(x)=\int_{0}^{\infty} \mathscr{F}(x) T_{2}(t) \phi_{3}(x) \mathrm{d} t
$$

to describe the total distribution of the new infective numbers produced during the infection period.

According to [14], the basic reproduction number is defined by $\mathfrak{R}_{0}=r(\mathscr{L})$, where $r(\mathscr{L})$ is the spectral radius of the operator $\mathscr{L}$. Furthermore, following Theorem 3 in [14], we have the following lemma.
Lemma 3. The principle eigenvalue $\lambda_{0}$ and $\mathfrak{R}_{0}-1$ have the same sign, and the disease-free steady state $E_{0}(x)$ is locally asymptotically stable.

\section{Extinction/Persistence Result}

Based on the discussion above, we now investigate the extinction and persistence of the disease.

Theorem 2. If $\mathfrak{R}_{0}<1$, then the disease-free steady state $E_{0}(x)$ is globally asymptotically stable.

Proof. By Lemma 2, we have $\lambda_{0}<0$ when $\mathfrak{R}_{0}<1$. Thus, there exists a small enough $\varepsilon>0$ such that $\lambda_{0}^{\varepsilon_{0}}<0$. According to Theorem 1 , there exists a $t_{*}>0$ such that $S_{i}(t, x) \leq S_{i}^{0}(x)+\varepsilon_{0}$ and $V_{i}(t, x) \leq V_{i}^{0}(x)+\varepsilon_{0}(1 \leq i \leq n)$ for all $x \in \Omega$. Thus, from the third equation of model (5) and assumption (4) that 


$$
\begin{cases}\frac{\partial I_{i}}{\partial t} \leq \nabla \cdot\left(d_{3 i}(x) \nabla I_{i}\right)+\sum_{j=1}^{n}\left(\beta_{i j}(x)\left(S_{i}^{0}(x)+\varepsilon_{0}\right)+\widetilde{\beta}_{i j}(x)\left(V_{i}^{0}(x)+\varepsilon_{0}\right)\right) f_{j}^{\prime}(0) I_{j}-\left(\mu_{i}^{I}(x)+\delta_{i}(x)+\gamma_{i}(x)\right) I_{i}, & x \in \Omega, t \geq t_{*}, 1 \leq i \leq n, \\ \frac{\partial I_{i}}{\partial \nu}=0, & x \in \partial \Omega, t \geq t_{*}, 1 \leq i \leq n,\end{cases}
$$

let $\left(\bar{\phi}_{31}(x), \bar{\phi}_{32}(x), \ldots, \bar{\phi}_{3 n}(x)\right)$ be the eigenfunction corresponding to the principal eigenvalue $\lambda_{0}^{\varepsilon_{0}}<0$. Assume that

$$
\left(I_{1}\left(t_{*}, x\right), I_{2}\left(t_{*}, x\right), \ldots, I_{n}\left(t_{*}, x\right)\right) \leq \alpha\left(\bar{\phi}_{31}(x), \bar{\phi}_{32}(x), \ldots, \bar{\phi}_{3 n}(x)\right)
$$

where $\alpha>0$ is a constant. With the aid of comparison principle, we can obtain

$$
\left.I(t, x)=\left(I_{1}(t, x), I_{2}(t, x), \ldots, I_{n}(t, x)\right) \leq \alpha\left(\bar{\phi}_{31}(x), \bar{\phi}_{32}(x), \ldots, \bar{\phi}_{3 n}(x)\right) e^{\lambda_{0}^{\varepsilon_{0}}\left(t-t_{*}\right.}\right), \quad t \geq t_{*} .
$$

This yields $\lim _{t \rightarrow \infty} I(t, x)=0$ uniformly for $x \in \bar{\Omega}$. Thus, the model (5) is asymptotic to (22). Then, by Lemma 2.2 in [40] and Corollary 4.3 in [46], we have $\lim _{t \rightarrow \infty} S(t, x)=S^{0}(x)$ and $\lim _{t \rightarrow \infty} V(t, x)=V^{0}(x)$.

Before proving the main results on disease persistence, we first need to establish the following lemma.

Lemma 4. Let $u(\cdot, t, \phi)=(S(\cdot, t, \phi), V(\cdot, t, \phi), I(\cdot, t, \phi))$ is the solution of model (5) with $u(\cdot, 0, \phi)=\phi=\left(\phi_{1}, \phi_{2}, \phi_{3}\right) \in \mathbb{X}^{+}$.

(i) For any $\phi \in \mathbb{X}^{+}$, we always have $S_{i}(\cdot, t, \phi)>0$ and $V_{i}(\cdot, t, \phi)>0$ for all $t>0$, and there exist constants $\rho_{1}>0, \rho_{2}>0$ such that

$$
\begin{gathered}
\liminf _{t \longrightarrow \infty} S_{i}(\cdot, t, \phi) \geq \rho_{1}, \\
\liminf _{t \rightarrow \infty} V_{i}(\cdot, t, \phi) \geq \rho_{2} .
\end{gathered}
$$

(ii) If there exists $t^{*} \geq 0$ such that $I_{i}\left(\cdot, t^{*}, \phi\right) \equiv 0$, then we have $I_{i}(\cdot, t, \phi)>0, \forall t>t^{*}, 1 \leq i \leq n$.

Proof. (i) It follows from Theorem 1 that there exists $t_{0}>0$ and $M>0$ such that

$$
I_{i}(\cdot, t, \phi) \leq M, \quad \forall t \geq t_{0} .
$$

Then, by the first equation of model (5) and the assumption (4), we have

$$
\begin{cases}\frac{\partial S_{i}}{\partial t} \geq \nabla \cdot\left(d_{1 i} \nabla S_{i}\right)+\lambda_{i}(x)-\left(\mu_{i}^{S}(x)+\xi_{i}(x)\right) S_{i}-\sum_{j=1}^{n} \beta_{i j}(x) M f_{j}^{\prime}(0) S_{i}, & x \in \Omega, t \geq t_{0} \\ \frac{\partial S_{i}}{\partial \nu}=0, & x \in \bar{\Omega}, t \geq t_{0} .\end{cases}
$$

Thus, by Lemma 2.2 in [40], it follows that the comparison system 


$$
\begin{cases}\frac{\partial w_{i}}{\partial t}=\nabla \cdot\left(d_{1 i} \nabla w_{i}\right)+\lambda_{i}(x)-\left(\mu_{i}^{S}(x)+\xi_{i}(x)\right) w_{i}-\sum_{j=1}^{n} \beta_{i j}(x) M f_{j}^{\prime}(0) w_{i}, & x \in \Omega, t \geq t_{0}, \\ \frac{\partial w_{i}}{\partial \nu}=0, & x \in \partial \Omega, t \geq t_{0},\end{cases}
$$

admits a unique positive steady state $w_{i}^{*}(x)$ which is globally asymptotically stable in $C(\bar{\Omega}, \mathbb{R})$. By the standard parabolic comparison theorem, there exists a constant $\rho_{1}>0$ such that $\liminf _{\mathrm{t} \longrightarrow \infty} S_{i}(\cdot, \mathrm{t}, \phi) \geq \rho_{1}$ is uniformly for $x \in \bar{\Omega}$. Moreover, it follows from the second equation of model (5) that

$$
\begin{cases}\frac{\partial V_{i}}{\partial t} \geq \nabla \cdot\left(d_{2 i} \nabla V_{i}\right)+\xi_{i}(x) \rho_{1}-\mu_{i}^{V}(x) V_{i}-\sum_{j=1}^{n} \widetilde{\beta}_{i j}(x) M f_{j}^{\prime}(0) V_{i}, & x \in \Omega, t \geq t_{0}, \\ \frac{\partial V_{i}}{\partial \nu}=0, & x \in \partial \Omega, t \geq t_{0} .\end{cases}
$$

Similarly, there exists a constant $\rho_{2}$ such that $\liminf _{\mathrm{t} \longrightarrow \infty} V_{i}(\cdot, \mathrm{t}, \phi) \geq \rho_{2}$ is uniformly for $x \in \bar{\Omega}$.

(ii) Assume $I_{i}\left(\cdot, t^{*}, \phi\right) \equiv 0$ for some $t^{*} \geq 0$. According to

Theorem 1, it follows from model (5) that

$$
\begin{cases}\frac{\partial I_{i}}{\partial t} \geq \nabla \cdot\left(d_{3 i} \nabla I_{i}\right)-\left(\mu_{i}^{I}(x)+\delta_{i}(x)+\gamma_{i}(x)\right) I_{i}, & x \in \Omega, t>0 \\ \frac{\partial I_{i}}{\partial v}=0, & x \in \partial \Omega, t>0\end{cases}
$$

Thus, the strong maximum principle (see, e.g., [47], Theorem 4) and Hopf boundary lemma (see, e.g., [47], Theorem 3$)$ imply that $I_{i}(\cdot, t, \phi)>0, \forall t>t^{*}$, and $x \in \bar{\Omega}$. Now, we are in position to state the main results of this section.
Theorem 3. If $\Re_{0}>1$, then there exists a constant $\varrho>0$ such that, for any $\phi=\left(\phi_{1}, \phi_{2}, \phi_{3}\right) \in \mathbb{X}^{+}$with $\phi_{3 i} \equiv 0(1 \leq i \leq n)$, solution $u(t, \cdot, \phi)=(S(t, \cdot, \phi), V(t, \cdot, \phi), I(t, \cdot, \phi))$ of model (5) satisfies

$$
\liminf _{t \longrightarrow \infty} S_{i}(t, \cdot, \phi) \geq \varrho, \liminf _{t \longrightarrow \infty} V_{i}(t, \cdot, \phi) \geq \varrho, \liminf _{t \longrightarrow \infty} I_{i}(t, \cdot, \phi) \geq \varrho, \quad 1 \leq i \leq n,
$$

uniformly for all $x \in \bar{\Omega}$. Moreover, model (5) has at least one endemic steady state $E_{*}(x)$.

Proof. Define

$$
\begin{aligned}
\mathbb{X}_{0} & =\left\{\phi=\left(\phi_{1}, \phi_{2}, \phi_{3}\right) \in \mathbb{X}^{+}: \phi_{3 i} \equiv 0, \quad 1 \leq i \leq n\right\}, \\
\partial \mathbb{X}_{0} & =\mathbb{X}^{+} \backslash \mathbb{X}_{0}=\left\{\phi=\left(\phi_{1}, \phi_{2}, \phi_{3}\right) \in \mathbb{X}^{+}: \phi_{3 i} \equiv 0, \quad 1 \leq i \leq n\right\} .
\end{aligned}
$$


According to Lemma 4, for any $\phi \in \mathbb{X}_{0}$, we have $I_{i}(t, \cdot, \phi)>0, \forall t>0$, and $x \in \bar{\Omega}$. Thus, $\Phi(t) \mathbb{X}_{0} \subseteq \mathbb{X}_{0}$, which implies that $\mathbb{X}_{0}$ is the invariant set for solution semiflow $\Phi(t)$ of model (5). Define

$$
\mathscr{M}_{\partial}=\left\{\phi \in \mathbb{X}^{+}: \Phi(t) \phi \in \partial \mathbb{X}_{0}, \quad \forall t \geq 0\right\},
$$

and $\omega(\phi)$ be the omega limit set of the orbit $\mathcal{O}^{+}(\phi)=\{\Phi(t) \phi: t \geq 0\}$. We first prove the following claim.

Claim 1. $\omega(\phi)=\left\{E_{0}\right\}=\left\{\left(S^{0}(x), V^{0}(x), 0\right)\right\}, \forall \phi \in \mathscr{M}_{\partial}$. Since $\phi \in \mathscr{M}_{\partial}$, we have $\Phi(t) \phi \in \partial \mathbb{X}_{0}$. Thus, $I_{i}(t, \cdot, \phi) \equiv 0(1 \leq i \leq n)$, $\forall t \geq 0$. Then, model (5) reduces to model (22); by Lemma 2.2 in [40] and Corollary 4.6 in [46], we have $\lim _{t \rightarrow \infty} S_{i}(t, \cdot, \phi)=S_{i}^{0}(x)$ and $\lim _{t \longrightarrow \infty} V_{i}(t, \cdot, \phi)=V_{i}^{0}(x)$ uniformly for $x \in \bar{\Omega}$. Hence, $\omega(\phi)=\left\{E_{0}\right\}=$ $\left\{\left(S^{0}(x), V^{0}(x), 0\right)\right\}, \forall \phi \in \mathscr{M}_{\partial}$; i.e., the claim holds.

Since $\mathfrak{R}_{0}>1$, we have $\lambda_{0}>0$ by Lemma 3 . Using the continuity of $\lambda_{0}$, there exists a sufficient small enough positive constant $\eta_{0}>0$ such that $\lambda_{0}^{\eta_{0}}>0$.
Claim 2. $E_{0}(x)$ is uniform weak repeller for $\mathbb{X}_{0}$ in the sense that

$$
\underset{t \rightarrow \infty}{\limsup }\left\|\Phi(t) \phi-E_{0}(x)\right\| \geq \eta_{0}, \quad \forall x \in \mathbb{X}_{0} .
$$

Suppose, by contradiction, there exists a $\phi_{0} \in \mathbb{X}_{0}$ such that

$$
\underset{t \rightarrow \infty}{\limsup }\left\|\Phi(t) \phi-E_{0}(x)\right\|<\eta_{0} .
$$

Then, there exists an enough large $t^{*}>0$ such that

$$
\begin{gathered}
S_{i}^{0}(x)-\eta_{0}<S_{i}(t, \cdot, \phi)<S_{i}^{0}(x)+\eta_{0}, V_{i}^{0}(x)-\eta_{0}<V_{i}(t, \cdot, \phi) \\
<V_{i}^{0}(x)+\eta_{0}, \quad 0<I_{i}(t, \cdot, \phi)<\eta_{0}, 1 \leq i \leq n, \forall t \geq t^{*}, x \in \bar{\Omega} .
\end{gathered}
$$

Therefore, from model (5) and assumptions (4), we have

$$
\begin{cases}\frac{\partial I_{i}}{\partial t} \geq \nabla \cdot\left(d_{3 i}(x) \nabla I_{i}\right)+\sum_{j=1}^{n}\left(\beta_{i j}(x)\left(S_{i}^{0}(x)-\eta_{0}\right)+\tilde{\beta}_{i j}(x)\left(V_{i}^{0}(x)-\eta_{0}\right)\right) f_{j}^{\prime}\left(\eta_{0}\right) I_{j}-\left(\mu_{i}^{I}(x)+\delta_{i}(x)+\gamma_{i}(x)\right) I_{i}, & x \in \Omega, t \geq t^{*}, 1 \leq i \leq n, \\ \frac{\partial I_{i}}{\partial \nu}=0, & x \in \partial \Omega, t \geq t^{*}, 1 \leq i \leq n .\end{cases}
$$

Set $\left(\varphi_{31}(x), \varphi_{32}(x), \ldots, \varphi_{3 n}(x)\right)$ be the strongly positive eigenfunction associated with principle eigenvalue $\lambda_{0}^{\eta_{0}}>0$. Since $I_{i}\left(t, \cdot, \phi_{0}\right)>0$ for all $x \in \bar{\Omega}$ and $t>0$, there exists a constant $\xi_{0}>0$ such that $I\left(t^{*}, \cdot, \phi_{0}\right) \geq \xi_{0}\left(\varphi_{31}(x), \varphi_{32}\right.$ $\left.(x), \ldots, \varphi_{3 n}(x)\right)$ for $x \in \bar{\Omega}$. It is clear that $\omega(t, x)=\xi_{0} e^{\lambda_{0}^{\eta_{0}}\left(t-t^{*}\right)}\left(\varphi_{31}(x), \varphi_{32}(x), \ldots, \varphi_{3 n}(x)\right)$ is a solution of the following linear system:

$$
\begin{cases}\frac{\partial \omega_{i}}{\partial t}=\nabla \cdot\left(d_{3 i}(x) \nabla \omega_{i}\right)+\sum_{j=1}^{n}\left(\beta_{i j}(x)\left(S_{i}^{0}(x)-\eta_{0}\right)+\tilde{\beta}_{i j}(x)\left(V_{i}^{0}(x)-\eta_{0}\right)\right) f_{j}^{\prime}\left(\eta_{0}\right) \omega_{j}-\left(\mu_{i}^{I}(x)+\delta_{i}(x)+\gamma_{i}(x)\right) \omega_{i}, & x \in \Omega, t \geq t^{*}, 1 \leq i \leq n, \\ \frac{\partial \omega_{i}}{\partial \nu}=0, & x \in \partial \Omega, t \geq t^{*}, 1 \leq i \leq n .\end{cases}
$$

Applying the comparison principle, we have

$$
\begin{array}{r}
I\left(t, \cdot, \phi_{0}\right) \geq \xi_{0} e^{\lambda_{0}^{\eta_{0}}\left(t-t^{*}\right)}\left(\varphi_{31}(x), \varphi_{32}(x), \ldots, \varphi_{3 n}(x)\right), \\
\forall x \in \bar{\Omega}, t \geq t^{*} .
\end{array}
$$

Due to $\lambda_{0}^{\eta_{0}}>0$, we conclude that $I_{i}\left(t, \cdot, \phi_{0}\right)$ is unbounded. This is a contradiction.

Define a continuous function $p: \mathbb{X}^{+} \longrightarrow[0,+\infty)$ by

$$
p(\phi)=\min \left\{\min _{x \in \bar{\Omega}} \phi_{3 i}(x)\right\}, \quad 1 \leq i \leq n, \forall \phi \in \mathbb{X}^{+} .
$$

Clearly, $p^{-1}(0,+\infty) \subseteq \mathbb{X}_{0}$ and has the property that if $p(\phi)>0$ or $p(\phi)=0$ and $\phi \in \mathbb{X}_{0}$, then $p(\Phi(t) \phi)>0$ for all $t>0$. Hence, for the semiflow $\Phi(t): \mathbb{X}^{+} \longrightarrow \mathbb{X}^{+}, p$ is a generalized distance function [48]. From Claim 1, it follows that any forward orbit of $\Phi(t)$ in $\mathscr{M}_{\partial}$ converges to $E_{0}(x)$. Moreover, Claim 2 implies that $E_{0}(x)$ is isolated in $\mathbb{X}^{+}$and $W^{s}\left(E_{0}(x)\right) \cap \mathbb{X}_{0}=\varnothing$, where $W^{s}\left(E_{0}(x)\right)$ is the stable set of $E_{0}(x)$. Furthermore, there is no cycle in $\mathscr{M}_{\partial}$ from $E_{0}(x)$ to $E_{0}(x)$. It then follows from Theorem 3 in [48] that there exists a constant $\varrho>0$ such that liminf $t \rightarrow \infty p(\Phi(t) \phi) \geq>$ for all $\phi \in \mathbb{X}_{0}$. This implies the uniform permanence of $I(t, \cdot, \phi)$. 
By Lemma 4 and Theorem 4.7 in [49], we know that $\Phi(t)$ has a positive steady state $E_{*}(x)$ of model (5). The proof is complete.

\section{Global Dynamics for a Special Case}

In this section, we consider a special case with all the parameters of model (5) as constants, except for the diffusion coefficients. Then, model (5) degenerates into the following model:

$$
\begin{cases}\frac{\partial S_{i}}{\partial t}=\nabla \cdot\left(d_{1 i}(x) \nabla S_{i}\right)+\lambda_{i}-\left(\mu_{i}^{S}+\xi_{i}\right) S_{i}-\sum_{j=1}^{n} \beta_{i j} S_{i} f_{j}\left(I_{j}\right), & t \geq 0, x \in \Omega, 1 \leq i \leq n \\ \frac{\partial V_{i}}{\partial t}=\nabla \cdot\left(d_{2 i}(x) \nabla V_{i}\right)+\xi_{i} S_{i}-\sum_{j=1}^{n} \widetilde{\beta}_{i j} V_{i} f_{j}\left(I_{j}\right)-\mu_{i}^{V} V_{i}, & t \geq 0, x \in \Omega, 1 \leq i \leq n, \\ \frac{\partial I_{i}}{\partial t}=\nabla \cdot\left(d_{3 i}(x) \nabla I_{i}\right)+\sum_{j=1}^{n}\left(\beta_{i j} S_{i}+\widetilde{\beta}_{i j} V_{i}\right) f_{j}\left(I_{j}\right)-\left(\mu_{i}^{I}+\delta_{i}+\gamma_{i}\right) I_{i}, & t \geq 0, x \in \Omega, 1 \leq i \leq n\end{cases}
$$

with the homogeneous Neumann boundary conditions (2) and the initial conditions (3). We mainly focus on the global stability of the endemic steady states of model (48). The proofs of the main results applying the Lyapunov functions and a subtle grouping technique guided by graph theory were developed in [28-30].

It follows from the Lemma 2 in [13] that model (48) has a disease-free steady state $E_{0}=\left(S^{0}, V^{0}, 0\right)$, where $S^{0}=\left(S_{1}^{0}, S_{2}^{0}, \ldots, S_{n}^{0}\right)$ and $V^{0}=\left(V_{1}^{0}, V_{2}^{0}, \ldots, V_{n}^{0}\right)$ with $S_{i}^{0}=\lambda_{i} / \mu_{i}^{S}+\xi_{i}$ and $V_{i}^{0}=\lambda_{i} \xi_{i} / \mu_{i}^{V}\left(\mu_{i}^{S}+\xi_{i}\right)$. Define matrices

$$
\begin{aligned}
& \mathscr{F}=\left(\begin{array}{cccc}
\theta_{11}^{0} f_{1}^{\prime}(0) & \theta_{12}^{0} f_{2}^{\prime}(0) & \ldots & \theta_{1 n}^{0} f_{n}^{\prime}(0) \\
\theta_{21}^{0} f_{1}^{\prime}(0) & \theta_{22}^{0} f_{2}^{\prime}(0) & \ldots & \theta_{2 n}^{0} f_{n}^{\prime}(0) \\
\vdots & \vdots & \ddots & \vdots \\
\theta_{n 1}^{0} f_{1}^{\prime}(0) & \theta_{n 2}^{0} f_{2}^{\prime}(0) & \ldots & \theta_{n n}^{0} f_{n}^{\prime}(0)
\end{array}\right), \\
& \mathscr{V}=\left(\begin{array}{cccc}
\mu_{1}^{I}+\delta_{1}+\gamma_{1} & 0 & \ldots & 0 \\
0 & \mu_{2}^{I}+\delta_{2}+\gamma_{2} & \ldots & 0 \\
\vdots & \vdots & \ddots & \vdots \\
0 & 0 & \ldots & \mu_{n}^{I}+\delta_{n}+\gamma_{n}
\end{array}\right),
\end{aligned}
$$

where $\theta_{i j}^{0}=\beta_{i j} S_{i}^{0}+\widetilde{\beta}_{i j} V_{i}^{0}$, then we have

$$
\mathscr{F} \mathscr{V}^{-1}=\left(\frac{\theta_{i j}^{0} f_{j}^{\prime}(0)}{\mu_{j}^{I}+\delta_{j}+\gamma_{j}}\right)_{1 \leq i, j \leq n} .
$$

Then, it follows from [26] that the basic reproduction number is defined as the spectral radius of $\mathscr{F} \mathscr{V}^{-1}$, i.e., $\mathfrak{R}_{0}=r\left(\mathscr{F} \mathscr{V}^{-1}\right)$. As a consequence of Theorems 2 and 3, we can obtain the following results without proofs.
Theorem 4. The disease-free steady state $E_{0}$ of model (48) is globally asymptotically stable when $\mathfrak{R}_{0}<1$.

Theorem 5. If $\mathfrak{R}_{0}>1$, then there exists a constant $\varrho>0$ such that, for any $\phi=\left(\phi_{1}, \phi_{2}, \phi_{3}\right) \in \mathbb{X}^{+}$with $\phi_{3 i} \equiv 0(1 \leq i \leq n)$, solution $u(t, \cdot, \phi)=(S(t, \cdot, \phi), V(t, \cdot, \phi), I(t, \cdot, \phi))$ of model (48) satisfies

$$
\begin{array}{r}
\liminf _{t \longrightarrow \infty} S_{i}(t, \cdot, \phi) \geq \varrho, \liminf _{t \longrightarrow \infty} V_{i}(t, \cdot, \phi) \geq \varrho, \liminf _{t \longrightarrow \infty} I_{i}(t, \cdot, \phi) \geq \varrho, \\
1 \leq i \leq n,
\end{array}
$$

uniformly for all $x \in \bar{\Omega}$. Moreover, model (48) has at least one endemic steady state.

Furthermore, set the endemic steady state $E_{*}=\left(S_{*}\right.$, $\left.V_{*}, I_{*}\right)$ with $S_{*}=\left(S_{1}^{*}, S_{2}^{*}, \ldots, S_{n}^{*}\right), V_{*}=\left(V_{1}^{*}, V_{2}^{*}, \ldots, V_{n}^{*}\right)$, and $I_{*}=\left(I_{1}^{*}, I_{2}^{*}, \ldots, I_{n}^{*}\right)$ satisfying

$$
\begin{cases}\lambda_{i}=\left(\mu_{i}^{S}+\xi_{i}\right) S_{i}^{*}+\sum_{j=1}^{n} \beta_{i j} S_{i}^{*} f_{j}\left(I_{j}^{*}\right), & 1 \leq i \leq n \\ \xi_{i} S_{i}^{*}=\sum_{j=1}^{n} \widetilde{\beta}_{i j} V_{i}^{*} f_{j}\left(I_{j}^{*}\right)+\mu_{i}^{V} V_{i}^{*}, & i \leq n, \\ \sum_{j=1}^{n}\left(\beta_{i j} S_{i}^{*}+\widetilde{\beta}_{i j} V_{i}^{*}\right) f_{j}\left(I_{j}^{*}\right)=\left(\mu_{i}^{I}+\delta_{i}+\gamma_{i}\right) I_{i}^{*}, & 1 \leq i \leq n .\end{cases}
$$

For the global stability of the endemic steady state $E_{*}$, we have the following conclusion.

Theorem 6. If $\mathfrak{R}_{0}>1$, then the endemic steady state $E_{*}$ is globally asymptotically stable. 
Proof. Define

$$
\begin{array}{r}
a_{i j}=\left(\beta_{i j} S_{i}^{*}+\widetilde{\beta}_{i j} V_{i}^{*}\right) f_{j}\left(I_{j}^{*}\right), \quad(1 \leq i, j \leq n), \\
\Theta=\left(\begin{array}{cccc}
\sum_{l \neq 1}^{n} a_{1 l} & -a_{21} & \ldots & -a_{n 1} \\
-a_{12} & \sum_{l \neq 2}^{n} a_{2 l} & \ldots & -a_{n 2} \\
\vdots & \vdots & \ddots & \vdots \\
-a_{1 n} & -a_{2 n} & \ldots & \sum_{l \neq n}^{n} a_{n l}
\end{array}\right),
\end{array}
$$

$$
H_{i}(t)=\int_{\Omega}\left[\left(S_{i}-S_{i}^{*}-S_{i}^{*} \ln \frac{S_{i}}{S_{i}^{*}}\right)+\left(V_{i}-V_{i}^{*}-V_{i}^{*} \ln \frac{V_{i}}{V_{i}^{*}}\right)+\left(I_{i}-I_{i}^{*}-I_{i}^{*} \ln \frac{I_{i}}{I_{i}^{*}}\right)\right] \mathrm{d} x
$$

Differentiating $H_{i}(t)$, we obtain

$$
\begin{aligned}
\frac{\mathrm{d} H_{i}(t)}{\mathrm{d} t}= & \int_{\Omega}\left\{\left(1-\frac{S_{i}}{S_{i}^{*}}\right)\left[\nabla \cdot\left(d_{1 i}(x) \nabla S_{i}\right)+\lambda_{i}-\sum_{j=1}^{n} \beta_{i j} S_{i} f_{j}\left(I_{j}\right)-\left(\mu_{i}^{S}+\xi_{i}\right) S_{i}\right]\right. \\
& +\left(1-\frac{V_{i}}{V_{i}^{*}}\right)\left[\nabla \cdot\left(d_{2 i}(x) \nabla V_{i}\right)+\xi_{i} S_{i}-\sum_{j=1}^{n} \widetilde{\beta}_{i j} V_{i} f_{j}\left(I_{j}\right)-\mu_{i}^{V} V_{i}\right]+\left(1-\frac{I_{i}}{I_{i}^{*}}\right) \\
& \left.\times\left[\nabla \cdot\left(d_{3 i}(x) \nabla I_{i}\right)+\sum_{j=1}^{n}\left(\beta_{i j} S_{i}+\widetilde{\beta}_{i j} V_{i}\right) f_{j}\left(I_{j}\right)-\left(\mu_{i}^{I}+\delta_{i}+\gamma_{i}\right) I_{i}\right]\right\} \mathrm{d} x .
\end{aligned}
$$

It follows from [50] that

$$
\begin{aligned}
\int_{\Omega} \nabla \cdot d_{1 i}(x) \nabla S_{i} \mathrm{~d} x & =0, \\
\int_{\Omega} \frac{1}{S_{i}} \nabla \cdot d_{1 i}(x) \nabla S_{i} \mathrm{~d} x & =\int_{\Omega} d_{1 i}(x) \frac{\left\|\nabla S_{i}\right\|^{2}}{S_{i}^{2}} \mathrm{~d} x, \\
\int_{\Omega} \nabla \cdot d_{2 i}(x) \nabla V_{i} \mathrm{~d} x & =0, \\
\int_{\Omega} \frac{1}{V_{i}} \nabla \cdot d_{2 i}(x) \nabla V_{i} \mathrm{~d} x & =\int_{\Omega} d_{2 i}(x) \frac{\left\|\nabla V_{i}\right\|^{2}}{V_{i}^{2}} \mathrm{~d} x, \\
\int_{\Omega} \nabla \cdot d_{3 i}(x) \nabla I_{i} \mathrm{~d} x & =0, \\
\int_{\Omega} \frac{1}{I_{i}} \nabla \cdot d_{3 i}(x) \nabla I_{i} \mathrm{~d} x & =\int_{\Omega} d_{3 i}(x) \frac{\left\|\nabla I_{i}\right\|^{2}}{I_{i}^{2}} \mathrm{~d} x .
\end{aligned}
$$


Then, using steady state equations (52), we have

$$
\begin{aligned}
& \frac{\mathrm{d} H_{i}(t)}{\mathrm{d} t}=\int_{\Omega}\left\{\mu_{i}^{S} S_{i}^{*}\left(2-\frac{S_{i}}{S_{i}^{*}}-\frac{S_{i}^{*}}{S_{i}}\right)+\mu_{i}^{V} V_{i}^{*}\left(3-\frac{V_{i}}{V_{i}^{*}}-\frac{S_{i}^{*}}{S_{i}}-\frac{S_{i} V_{i}^{*}}{S_{i}^{*} V_{i}}\right)+\sum_{j=1}^{n} \beta_{i j} S_{i}^{*} f_{j}\left(I_{j}^{*}\right)\left(2-\frac{S_{i}^{*}}{S_{i}}-\frac{I_{i}}{I_{i}^{*}}+\frac{f_{j}\left(I_{j}\right)}{f_{j}\left(I_{j}^{*}\right)}-\frac{S_{i} I_{i}^{*} f_{j}\left(I_{j}\right)}{S_{i}^{*} I_{i} f_{j}\left(I_{j}^{*}\right)}\right)\right. \\
& +\sum_{j=1}^{n} \tilde{\beta}_{i j} V_{i}^{*} f_{j}\left(I_{j}^{*}\right)\left(3-\frac{S_{i}^{*}}{S_{i}}-\frac{I_{i}}{I_{i}^{*}}+\frac{f_{j}\left(I_{j}\right)}{f_{j}\left(I_{j}^{*}\right)}-\frac{S_{i} V_{i}^{*}}{S_{i}^{*} V_{i}}-\frac{V_{i} I_{i}^{*} f_{j}\left(I_{j}\right)}{V_{i}^{*} I_{i} f_{j}\left(I_{j}^{*}\right)}\right)-d_{1 i}(x) S_{i}^{*} \frac{\left\|\nabla S_{i}\right\|^{2}}{S_{i}^{2}}-d_{2 i}(x) V_{i}^{*} \frac{\left\|\nabla V_{i}\right\|^{2}}{V_{i}^{2}} \\
& \left.-d_{3 i}(x) I_{i}^{*} \frac{\left\|\nabla I_{i}\right\|^{2}}{I_{i}^{2}}\right\} \mathrm{d} x \\
& =\int_{\Omega}\left\{\mu_{i}^{S} S_{i}^{*}\left(2-\frac{S_{i}}{S_{i}^{*}}-\frac{S_{i}^{*}}{S_{i}}\right)+\mu_{i}^{V} V_{i}^{*}\left(3-\frac{V_{i}}{V_{i}^{*}}-\frac{S_{i}^{*}}{S_{i}}-\frac{S_{i} V_{i}^{*}}{S_{i}^{*} V_{i}}\right)+\sum_{j=1}^{n} \beta_{i j} S_{i}^{*} f_{j}\left(I_{j}^{*}\right)\right. \\
& \cdot\left[\varphi\left(\frac{S_{i}^{*}}{S_{i}}\right)+\varphi\left(\frac{S_{i} I_{i}^{*} f_{j}\left(I_{j}\right)}{S_{i}^{*} I_{i} f_{j}\left(I_{j}^{*}\right)}\right)+\varphi\left(\frac{I_{i} f_{i}\left(I_{i}^{*}\right)}{I_{i}^{*} f_{i}\left(I_{i}\right)}\right)+\left(\frac{f_{i}\left(I_{i}\right)}{f_{i}\left(I_{i}^{*}\right)}-\frac{I_{i}}{I_{i}^{*}}\right)\left(1-\frac{f_{i}\left(I_{i}^{*}\right)}{f_{i}\left(I_{i}\right)}\right)\right]+\frac{f_{j}\left(I_{j}\right)}{f_{j}\left(I_{j}^{*}\right)}-\frac{f_{i}\left(I_{i}\right)}{f_{i}\left(I_{i}^{*}\right)}+\ln \frac{f_{j}\left(I_{j}^{*}\right) f_{i}\left(I_{i}\right)}{f_{j}\left(I_{j}\right) f_{i}\left(I_{i}^{*}\right)} \\
& +\sum_{j=1}^{n} \widetilde{\beta}_{i j} V_{i}^{*} f_{j}\left(I_{j}^{*}\right)\left[\varphi\left(\frac{S_{i}^{*}}{S_{i}}\right)+\varphi\left(\frac{S_{i} V_{i}^{*}}{S_{i}^{*} V_{i}}\right)+\varphi\left(\frac{V_{i} I_{i}^{*} f_{j}\left(I_{j}\right)}{V_{i}^{*} I_{i} f_{j}\left(I_{j}^{*}\right)}\right)+\varphi\left(\frac{I_{i} f_{i}\left(I_{i}^{*}\right)}{I_{i}^{*} f_{i}\left(I_{i}\right)}\right)+\left(\frac{f_{i}\left(I_{i}\right)}{f_{i}\left(I_{i}^{*}\right)}-\frac{I_{i}}{I_{i}^{*}}\right)\left(1-\frac{f_{i}\left(I_{i}^{*}\right)}{f_{i}\left(I_{i}\right)}\right)\right. \\
& \left.\left.+\frac{f_{j}\left(I_{j}\right)}{f_{j}\left(I_{j}^{*}\right)}-\frac{f_{i}\left(I_{i}\right)}{f_{i}\left(I_{i}^{*}\right)}+\ln \frac{f_{j}\left(I_{j}^{*}\right) f_{i}\left(I_{i}\right)}{f_{j}\left(I_{j}\right) f_{i}\left(I_{i}^{*}\right)}\right]-d_{1 i}(x) S_{i}^{*} \frac{\left\|\nabla S_{i}\right\|^{2}}{S_{i}^{2}}-d_{2 i}(x) V_{i}^{*} \frac{\left\|\nabla V_{i}\right\|^{2}}{V_{i}^{2}}-d_{3 i}(x) I_{i}^{*} \frac{\left\|\nabla I_{i}\right\|^{2}}{I_{i}^{2}}\right\} \mathrm{d} x,
\end{aligned}
$$

where $\varphi(x)=1+\ln x-x$ with global maximum value $\varphi(x)=0$. From the assumption (4), it is easy to validate that

$$
\left(\frac{f_{i}\left(I_{i}\right)}{f_{i}\left(I_{i}^{*}\right)}-\frac{I_{i}}{I_{i}^{*}}\right)\left(1-\frac{f_{i}\left(I_{i}^{*}\right)}{f_{i}\left(I_{i}\right)}\right) \leq 0 .
$$

Moreover, according to the property that arithmetic mean is not less than the associated geometric mean, $V_{i} / V_{i}^{*}+S_{i}^{*} / S_{i}+S_{i} V_{i}^{*} / S_{i}^{*} V_{i} \geq 3$ and $S_{i} / S_{i}^{*}+S_{i}^{*} / S_{i} \geq 2$. Thus, we have

$$
\frac{\mathrm{d} H_{i}(t)}{\mathrm{d} t} \leq \int_{\Omega}\left[\sum_{j=1}^{n}\left(\beta_{i j} S_{i}^{*}+\widetilde{\beta}_{i j} V_{i}^{*}\right) f_{j}\left(I_{j}^{*}\right)\left(\frac{f_{j}\left(I_{j}\right)}{f_{j}\left(I_{j}^{*}\right)}-\frac{f_{i}\left(I_{i}\right)}{f_{i}\left(I_{i}^{*}\right)}+\ln \frac{f_{j}\left(I_{j}^{*}\right) f_{i}\left(I_{i}\right)}{f_{j}\left(I_{j}\right) f_{i}\left(I_{i}^{*}\right)}\right)\right] \mathrm{d} x .
$$

Consequently, we obtain

$$
\frac{\mathrm{d} \Phi(t)}{\mathrm{d} t} \leq \int_{\Omega} \sum_{i=1}^{n} \zeta_{i}\left[\sum_{j=1}^{n}\left(\beta_{i j} S_{i}^{*}+\tilde{\beta}_{i j} V_{i}^{*}\right) f_{j}\left(I_{j}^{*}\right)\left(\frac{f_{j}\left(I_{j}\right)}{f_{j}\left(I_{j}^{*}\right)}-\frac{f_{i}\left(I_{i}\right)}{f_{i}\left(I_{i}^{*}\right)}+\ln \frac{f_{j}\left(I_{j}^{*}\right) f_{i}\left(I_{i}\right)}{f_{j}\left(I_{j}\right) f_{i}\left(I_{i}^{*}\right)}\right)\right] \mathrm{d} x .
$$


It follows from the equality $\Theta \zeta=0$ that $\sum_{j=1}^{n} a_{j i} \zeta_{j}=$ $\sum_{l=1}^{n} a_{i l} \zeta_{i}$ which is equivalent to $\sum_{j=1}^{n}\left(\beta_{j i} S_{j}^{*}+\widetilde{\beta}_{j i} V_{j}^{*}\right) f_{i}\left(I_{i}^{*}\right)$ $\zeta_{j}=\sum_{l=1}^{n}\left(\beta_{i l} S_{i}^{*}+\tilde{\beta}_{i l} V_{i}^{*}\right) f_{l}\left(I_{l}^{*}\right) \zeta_{i}$. Then,

$$
\begin{aligned}
\sum_{i=1}^{n} \sum_{j=1}^{n}\left(\beta_{i j} S_{i}^{*}+\tilde{\beta}_{i j} V_{i}^{*}\right) f_{j}\left(I_{j}\right) \zeta_{i} & =\sum_{i=1}^{n} \sum_{j=1}^{n}\left(\beta_{j i} S_{j}^{*}+\tilde{\beta}_{j i} V_{j}^{*}\right) f_{i}\left(I_{i}\right) \zeta_{j} \\
& =\sum_{i=1}^{n} \frac{f_{i}\left(I_{i}\right)}{f_{i}\left(I_{i}^{*}\right)} \sum_{j=1}^{n}\left(\beta_{j i} S_{j}^{*}+\widetilde{\beta}_{j i} V_{j}^{*}\right) f_{i}\left(I_{i}^{*}\right) \zeta_{j}=\sum_{i=1}^{n} \frac{f_{i}\left(I_{i}\right)}{f_{i}\left(I_{i}^{*}\right)} \sum_{l=1}^{n}\left(\beta_{i l} S_{i}^{*}+\tilde{\beta}_{i l} V_{i}^{*}\right) f_{l}\left(I_{l}^{*}\right) \zeta_{i} \\
& =\sum_{i=1}^{n} \sum_{j=1}^{n}\left(\beta_{i j} S_{i}^{*}+\tilde{\beta}_{i j} V_{i}^{*}\right) \frac{f_{i}\left(I_{i}\right)}{f_{i}\left(I_{i}^{*}\right)} f_{j}\left(I_{j}^{*}\right) \zeta_{i},
\end{aligned}
$$

and thus, $\sum_{i=1}^{n} \sum_{j=1}^{n}\left(\beta_{i j} S_{i}^{*}+\widetilde{\beta}_{i j} V_{i}^{*}\right) f_{j}\left(I_{j}^{*}\right)\left(f_{j}\left(I_{j}\right) / f_{j}\left(I_{j}^{*}\right)-\right.$ $\left.f_{i}\left(I_{i}\right) / f_{i}\left(I_{i}^{*}\right)\right)=0$ for all $I_{1}, I_{2}, \ldots, I_{n}>0$. Following the graph-theoretic approach as proposed in [28-30], similar procedures as in [26] can be applied to verify that

$$
\sum_{i=1}^{n} \zeta_{i} \sum_{j=1}^{n}\left(\beta_{i j} S_{i}^{*}+\widetilde{\beta}_{i j} V_{i}^{*}\right) f_{j}\left(I_{j}^{*}\right) \ln \frac{f_{j}\left(I_{j}^{*}\right) f_{i}\left(I_{i}\right)}{f_{j}\left(I_{j}\right) f_{i}\left(I_{i}^{*}\right)}=0 .
$$

Thus, we have $\mathrm{d} \Phi(t) / \mathrm{d} t \leq 0$. Furthermore, it can be shown that the largest invariant set in $\{(S, V, I): \mathrm{d} \Phi(t) / \mathrm{d} t=$ $0\}$ is the singleton $\left\{E_{*}\right\}$. Therefore, by the LaSalle's invariance principle, $E_{*}$ is globally asymptotically stable.

\section{Conclusions}

In this paper, a multigroup SVIR model with diffusion and nonlinear incidence rate has been investigated. We defined the basic reproduction number $\mathfrak{R}_{0}$ for spatially heterogeneous environment, and we further proved that $\mathfrak{R}_{0}$ served as a threshold index which predicts the extinction and persistence of the disease. Particularly, by using comparison principle, we proved that the diseasefree steady state $E_{0}(x)$ is globally asymptotically stable when $\mathfrak{R}_{0}$ is less than one. If $\boldsymbol{R}_{0}$ is great than one, then the disease will persist. Consequently, we obtained the existence of endemic steady state $E_{*}(x)$. Furthermore, we established the criteria on the global stability of the disease-free steady state and the endemic steady state of the model in a special case.

Although the existence of the endemic steady state is established, it is necessary to point that the uniqueness and stability of the endemic steady state remains an open problem. Moreover, the impact of the latent individuals should also be considered for some diseases during its spread, such as AIDS and COVID-19 (see, for example, $[4-8,51])$, in which it has been validated that the latent individuals for these two diseases may also have probability of infection. Thus, an improved model with latent compartment should be investigated. We leave these problems for future investigation.

\section{Data Availability}

There are no data in the submission.

\section{Conflicts of Interest}

The authors declare that there are no conflicts of interest.

\section{Acknowledgments}

This work was supported by the National Natural Science Foundation of China (11701445, 11701451, and 11702214), Natural Science Basic Research Plan in Shaanxi Province of China (2018JQ1057 and 2020JQ-38), and Young Talent Fund of University Association for Science and Technology in Shaanxi, China (20180504).

\section{References}

[1] A. G. McKendrick, "Applications of mathematics to medical problems," Proceedings of the Edinburgh Mathematical Society, vol. 44, pp. 98-130, 1925.

[2] O. Diekmann and J. A. P. Heesterbeek, Mathematical Epidemiology of Infectious Diseases, Wiley, Chichester, UK, 2000.

[3] X. Liu, Y. Takeuchi, and S. Iwami, "SVIR epidemic models with vaccination strategies," Journal of Theoretical Biology, vol. 253, no. 1, pp. 1-11, 2008.

[4] L. Wang, J. Wang, J. Wang et al., "Modelling and assessing the effects of medical resources on transmission of novel coronavirus (COVID-19) in Wuhan, China," Mathematical Biosciences and Engineering, vol. 17, no. 4, pp. 2936-2949, 2020.

[5] J. Jiao, Z. Liu, and S. Cai, "Dynamics of an SEIR model with infectivity in incubation period and homestead-isolation on the susceptible," Applied Mathematics Letters, vol. 107, Article ID 106442, 2020.

[6] W. Zhou, A. Wang, F. Xia et al., "Effects of media reporting on mitigating spread of COVID-19 in the early phase of the outbreak," Math. Biosci. Eng.vol. 17, no. 3, pp. 2683-2707, 2020.

[7] B. Tang, F. Xia, S. Tang et al., "The effectiveness of quarantine and isolation determine the trend of the COVID-19 epidemics in the final phase of the current outbreak in China," 
International Journal of Infectious Diseases, vol. 95, pp. 288293, 2020.

[8] B. Tang, F. Xia, S. Tang et al., "The evolution of quarantined and suspected cases determines the final trend of the 2019nCoV epidemics based on multi-source data analyses," 2020, https://ssrn.com/abstract=3537099.

[9] L. Peng, W. Yang, D. Zhang et al., "Epidemic analysis of COVID-19 in China by dynamical modeling," 2020, http:// arxiv.org/abs/2002.06563.

[10] B. Tang, X. Wang, Q. Li et al., "Estimation of the transmission risk of the 2019-nCoV and its implication for public health interventions," Journal of Clinical Medicine, vol. 9, no. 2, 2020.

[11] J. Wang and J. Wang, "Analysis of a reaction-diffusion cholera model with distinct dispersal rates in the human population," Journal of Dynamics and Differential Equations, pp. 1-27, 2020.

[12] R. Peng and X.-Q. Zhao, "A reaction-diffusion SIS epidemic model in a time-periodic environment," Nonlinearity, vol. 25, no. 5, pp. 1451-1471, 2012.

[13] Y. Lou and X.-Q. Zhao, "A reaction-diffusion malaria model with incubation period in the vector population," Journal of Mathematical Biology, vol. 62, no. 4, pp. 543-568, 2011.

[14] W. Wang and X.-Q. Zhao, "Basic reproduction numbers for reaction-diffusion epidemic models," SIAM Journal on Applied Dynamical Systems, vol. 11, no. 4, pp. 1652-1673, 2012.

[15] Y. Wu and X. Zou, "Asymptotic profiles of steady states for a diffusive SIS epidemic model with mass action infection mechanism," Journal of Differential Equations, vol. 261, no. 8, pp. 4424-4447, 2016.

[16] T. Kuniya and J. Wang, "Global dynamics of an SIR epidemic model with nonlocal diffusion," Nonlinear Analysis: Real World Applications, vol. 43, pp. 262-282, 2018.

[17] Y. Cai, Y. Kang, M. Banerjee, and W. Wang, "Complex dynamics of a host-parasite model with both horizontal and vertical transmissions in a spatial heterogeneous environment," Nonlinear Analysis: Real World Applications, vol. 40, pp. 444-465, 2018.

[18] Z. Bai, R. Peng, and X.-Q. Zhao, "A reaction-diffusion malaria model with seasonality and incubation period," Journal of Mathematical Biology, vol. 77, no. 1, pp. 201-228, 2018.

[19] X. Ren, Y. Tian, L. Liu, and X. Liu, "A reaction-diffusion withinhost HIV model with cell-to-cell transmission," Journal of Mathematical Biology, vol. 76, no. 7, pp. 1831-1872, 2018.

[20] Y.-C. Shyu, R.-N. Chien, and F.-B. Wang, "Global dynamics of a West Nile virus model in a spatially variable habitat," Nonlinear Analysis: Real World Applications, vol. 41, pp. 313-333, 2018.

[21] Z. Xu, Y. Xu, and Y. Huang, "Stability and traveling waves of a vaccination model with nonlinear incidence," Computers \& Mathematics with Applications, vol. 75, no. 2, pp. 561-581, 2018.

[22] R. Zhang, S. Liu, and S. Liu, "Traveling waves for SVIR epidemic model with nonlocal dispersal," Mathematical Biosciences and Engineering, vol. 16, no. 3, pp. 1654-1682, 2019.

[23] A. Lajmanovich and J. A. Yorke, "A deterministic model for gonorrhea in a nonhomogeneous population," Math. Biosci.vol. 28, no. 3-4, pp. 221-236, 1976.

[24] Y. Muroya, Y. Enatsu, and T. Kuniya, "Global stability for a multi-group SIRS epidemic model with varying population sizes," Nonlinear Analysis: Real World Applications, vol. 14, no. 3, pp. 1693-1704, 2013.

[25] T. Kuniya, "Global stability of a multi-group SVIR epidemic model," Nonlinear Analysis: Real World Applications, vol. 14, no. 2, pp. 1135-1143, 2013.
[26] Y. Geng and J. Xu, "Stability preserving NSFD scheme for a multi-group SVIR epidemic model," Mathematical Methods in the Applied Sciences, vol. 40, no. 13, pp. 4917-4927, 2017.

[27] H. Shu, D. Fan, and J. Wei, "Global stability of multi-group SEIR epidemic models with distributed delays and nonlinear transmission," Nonlinear Analysis: Real World Applications, vol. 13, no. 4, pp. 1581-1592, 2012.

[28] H. Guo, M. Y. Li, and Z. Shuai, "A graph-theoretic approach to the method of global Lyapunov functions," Proceedings of the American Mathematical Society, vol. 136, no. 08, pp. 2793-2802, 2008.

[29] H. Guo, M. Y. Li, and Z. Shuai, "Global stability of the endemic equilibrium of multigroup SIR epidemic models," Canadian Applied Mathematics Quarterly, vol. 14, pp. 259284, 2006.

[30] M. Y. Li and Z. Shuai, "Global-stability problem for coupled systems of differential equations on networks," Journal of Differential Equations, vol. 248, no. 1, pp. 1-20, 2010.

[31] S.-L. Wu and G. Chen, "Uniqueness and exponential stability of traveling wave fronts for a multi-type SIS nonlocal epidemic model," Nonlinear Analysis: Real World Applications, vol. 36, pp. 267-277, 2017.

[32] S.-L. Wu and P. Weng, "Entire solutions for a multi-type SIS nonlocal epidemic model inRorZ , Z," Journal of Mathematical Analysis and Applications, vol. 394, no. 2, pp. 603-615, 2012.

[33] S.-L. Wu, P. Li, and H. Cao, "Dynamics of a nonlocal multitype SIS epidemic model with seasonality," Journal of Mathematical Analysis and Applications, vol. 463, no. 1, pp. 111-133, 2018.

[34] Y. Luo, S. Tang, Z. Teng, and L. Zhang, "Global dynamics in a reaction-diffusion multi-group SIR epidemic model with nonlinear incidence," Nonlinear Analysis: Real World Applications, vol. 50, pp. 365-385, 2019.

[35] G. Huang, Y. Takeuchi, W. Ma, and D. Wei, "Global stability for delay SIR and SEIR epidemic models with nonlinear incidence rate," Bulletin of Mathematical Biology, vol. 72, no. 5, pp. 1192-1207, 2010.

[36] A. Korobeinikov and P. K. Maini, "Non-linear incidence and stability of infectious disease models," Mathematical Medicine and Biology: A Journal of the IMA, vol. 22, no. 2, pp. 113-128, 2005.

[37] Y. Yang, J. Zhou, and C.-H. Hsu, "Threshold dynamics of a diffusive SIRI model with nonlinear incidence rate," Journal of Mathematical Analysis and Applications, vol. 478, no. 2, pp. 874-896, 2019.

[38] H. L. Smith, "Monotone dynamical systems: an introduction to the theory of competitive and cooperative systems," in Mathematical Surveys and MonographsVol. 41, American Mathematical Society, Providence, RI, 1995.

[39] R. H. Martin and H. L. Smith, "Abstract functional differential equations and reaction-diffusion systems," Transactions of the American Mathematical Society, vol. 321, no. 1, pp. 1-44, 1990.

[40] Z. Guo, F.-B. Wang, and X. Zou, "Threshold dynamics of an infective disease model with a fixed latent period and nonlocal infections," Journal of Mathematical Biology, vol. 65, no. 6-7, pp. 1387-1410, 2012.

[41] Y. Du, "Order structure and topological methods in nonlinear partial differential equations," in Maximum Principles and ApplicationsVol. 1, World Scientific, New Jersey, NJ, USA, 2006.

[42] R. B. Guenther and J. W. Lee, Partial Differential Equations of Mathematical Physics and Integral Equations, Dover. Public. Inc., Mineola, NY, USA, 1996. 
[43] M. Wang, Nonlinear Elliptic Equations, Science Public., Beijing, China, 2010.

[44] J. Wu, Theory and Applications of Partial Functional Differential Equations, Springer-Verlag, New York, NY, USA, 1996.

[45] J. K. Hale, Asymptotic Behavior of Dissipative Systems, American Mathematical Society, Providence, RI, USA, 1988.

[46] H. R. Thieme, "Convergence results and a Poincar $e$-Bendixson trichotomy for asymptotically autonomous differential equations," J. Math. Biol.vol. 30, pp. 755-763, 1992.

[47] M. H. Protter and H. F. Weinberger, Maximum Principles in Differential Equations, Springer-Verlag, New York, NY, USA, 1984.

[48] H. L. Smith and X.-Q. Zhao, "Robust persistence for semidynamical systems," Nonlinear Analysis: Theory, Methods \& Applications, vol. 47, no. 9, pp. 6169-6179, 2001.

[49] P. Magal and X.-Q. Zhao, "Global attractors and steady states for uniformly persistent dynamical systems," SIAM Journal on Mathematical Analysis, vol. 37, no. 1, pp. 251-275, 2005.

[50] J. Groeger, "Divergence theorems and the supersphere," Journal of Geometry and Physics, vol. 77, pp. 13-29, 2014.

[51] H.-F. Huo and L.-X. Feng, "Global stability for an HIV/AIDS epidemic model with different latent stages and treatment," Applied Mathematical Modelling, vol. 37, no. 3, pp. 14801489, 2013. 